\title{
A 2.5D Computational Method to Simulate Cylindrical Fluidized Beds
}

Tingwen $\mathrm{Li}^{\mathrm{a}, \mathrm{b},{ }^{*}}$, Sofiane Benyahia ${ }^{\mathrm{a}}$, Jean-François Dietiker ${ }^{\mathrm{a}, \mathrm{c}}$, Jordan Musser ${ }^{\mathrm{a}}$, Xin Sun ${ }^{\mathrm{d}}$

a National Energy Technology Laboratory, Morgantown, WV 26505, USA

b URS Corporation, Morgantown, WV 26505, USA

c West Virginia University Research Corporation, Morgantown, WV 26506, USA

d Pacific Northwest National Laboratory, Richland, WA 99354, USA

\begin{abstract}
In this paper, the limitations of axisymmetric and Cartesian two-dimensional (2D) simulations of cylindrical gas-solid fluidized beds are discussed. A new method has been proposed to carry out pseudo-two-dimensional (2.5D) simulations of a cylindrical fluidized bed by appropriately combining the benefits of Cartesian 2D and axisymmetric assumptions. This is done by constructing a computational domain consisting of a central thin plate and two wedges. The proposed method was implemented in the open-source code MFIX and applied to the simulation of a lab-scale bubbling fluidized bed with necessary sensitivity study. After a careful grid study to ensure the numerical results are grid independent, detailed comparisons of the flow hydrodynamics were presented against axisymmetric and Cartesian 2D simulations. Furthermore, the 2.5D simulation results have been compared to the three-dimensional (3D) simulation for evaluation. This new approach yields better agreement with the 3D simulation results than with axisymmetric and Cartesian 2D simulations.
\end{abstract}

Keyword: Computational fluid dynamics, fluidized bed, two-fluid model, twodimensional simulation.

* Corresponding author at: National Energy Technology Laboratory, Morgantown, WV 26505, USA. Tel.: 13042854538.

E-mail addresses: tingwen.li@contr.netl.doe.gov, litingwen@gmail.com (T. Li). 


\section{Introduction}

A gas-solids fluidized bed is a complex system with multi-scale temporal and spatial variations. With the rapid development of high-performance computers, computational algorithms, and multiphase flow models, computational fluid dynamic (CFD) modeling has become an effective tool to help researchers better understand the complex flow hydrodynamics in fluidized beds. However, the computational cost of simulating gassolids flows is extremely large due to inherent unsteady and highly coupled multi-scale characteristics that require highly resolved numerical grids and small time steps. The numerical simulations of real fluidized bed systems, which in industry are typically cylindrical columns, consume significant time and resources. To alleviate the computational cost of transient simulations of gas-solids cylindrical fluidized beds, numerous qualitative/quantitative two-dimensional (2D) numerical studies, with axisymmetric assumption or 2D Cartesian assumption, have been reported in the literature for most flow regimes, e.g. bubbling, slugging, turbulent, and circulating fluidized beds.

Considering the symmetry in geometry and boundary conditions of most cylindrical gassolids fluidized beds in batch mode operation, it is natural to make the axisymmetric assumption to manage the computational cost. This assumption has been extensively used in experiments to reduce the number of data points for measurement as the mean flow field demonstrates good symmetry about the central axis (Drake and Heindel 2011; Weber and Mei 2013). Axisymmetrical simulations of bubbling fluidized beds have been reported by several studies with certain successes (Pain et al. 2002; Lindborg et al. 2007). However, it has been generally observed that the axisymmetric assumption prevents the gas-solids flow from crossing the central axis, which behaves as a free-slip wall (Pain et al. 2001; Cabezas-Gomez and Milioli 2003; Reuge et al. 2008; Xie et al. 2008a). High solid concentration and downward solids flow tend to be predicted along the central axis of the bubbling fluidized bed, which is inconsistent with the experimental observation of the usually upward flow with high voidage in the central region. To overcome this limitation and to allow particles to cross the central axis, Sun and Gidaspow (1999) solved the governing equations over a full 2D plane in cylindrical coordinates without assuming symmetry along the axis. The singularity at the central axis $(r=0)$ in cylindrical coordinates was overcome by carefully setting up the numerical mesh to avoid the grid point at $r=0$ in their finite difference CFD code. With this method, they reported reasonably good agreement between numerical prediction and experimental measurement for a circulating fluidized bed riser flow. However, no other work in the literature can be found using this method for the gas-solids fluidized bed modeling. 
Acknowledging the unphysical accumulation of solids along the central line associated with the axisymmetric flow assumption, the Cartesian 2D simulation along a central plane is commonly used to simulate the gas-solids fluidized bed. Compared to the axisymmetric simulation, the Cartesian 2D simulation breaks the symmetry constraint along the central axis and predicts qualitatively consistent results with the experimental observation. Hence, the 2D Cartesian approximation to a 3D cylindrical bed is usually preferred when 3D simulation of such system is infeasible. Extensive numerical studies of various fluidized bed systems using the Cartesian 2D simulations can be found in the open literature.

Although the 2D Cartesian simulations have been widely used for both qualitative and quantitative investigation of various gas-solids fluidized bed systems, certain differences between the Cartesian 2D simulation and the 3D simulation are generally acknowledged. The differences between 2D and 3D simulations of gas-solids fluidized beds and the applicability of the 2D Cartesian model have been discussed by several authors (Peirano et al. 2001; Cammarata et al. 2003; Xie et al. 2008a, 2008b; Li et al. 2010; Cloete et al. 2013; Li et al. 2014a, 2014b). The 2D simulation clearly fails to capture the inherent three-dimensionality in the gas-solids flow such as clusters and bubbles. In addition, a 2D numerical simulation is not capable of accurately accounting for the 3D effects resulting from the boundary conditions imposed by the column wall. Generally, all these studies suggest that significant quantitative differences exist between 2D and 3D simulations, therefore the 2D simulation should only be used for qualitative studies. The reported differences are attributed to the inherent three-dimensional nature of gas-solids flows and the geometrical inconsistency of simplifying a cylinder to a Cartesian 2D plane.

In this paper, a novel pseudo-2D computational domain (referred to as "2.5D" hereafter) is proposed to combine the Cartesian 2D assumption with the axisymmetric flow assumption to model a cylindrical fluidized bed. In this method, a novel computational domain, i.e., two wedges connected by a thin plate, is proposed to take advantage of the benefits from both assumptions. The 2.5D model is tested for a lab-scale fluidized bed system as reported by Reuge et al. (2008). A series of simulations were conducted using different flow assumptions. Comparisons of the new model results with the axisymmetric simulation and the Cartesian 2D simulation of the same system are made. Finally, the new method is verified against the most accurate (and expensive) 3D simulations. 


\section{Model Description}

The mean flow field is assumed to be axisymmetric for a typical cylindrical gas-solids fluidized bed operated under the batch mode with fluidizing gas uniformly fed through the bottom distributor. This is a valid assumption as confirmed by numerous experimental observations of time-averaged flow fields, although instantaneous flow behavior does not show such symmetry (e.g. Zhang et al. 1991; Drake and Heindel 2011; Weber and Mei 2013). This assumption is also applicable to a system with an asymmetric configuration, such as a side-inlet in a circulating fluidized bed, which has limited effect on the bulk flow behavior (e.g. Malcus et al. 2000, Du et al. 2005). Figure 1 schematically illustrates the axisymmetric and 2D Cartesian simulations of a cylindrical fluidized bed. The axisymmetric and 2D Cartesian simulations are equivalent to 3D simulations of the flow in a wedge and plane with unit arc and thickness, discretized by a single layer of computational cells. Limited by the computational domain, the axisymmetric 2D simulation does not allow the flow to pass the central axis, while the 2D Cartesian simulation breaks the geometry similarity to the cylindrical system. Both assumptions have been discussed in the literature.

Figure 1.

A novel computational domain made of two wedges connected by a thin plate, as schematically shown in Figure 2, is proposed to combine the advantages of axisymmetric and 2D Cartesian simulations. Here, the ratio between the plate half width, $L$, and the wedge radius, $R$, is adjustable and determines the thickness of the plate, $H$. For a very small $L / R$, the domain becomes axisymmetric. While for $L / R$ close to unity, the simulation becomes two-dimensional. This approach attempts to impose flow symmetry in a cylindrical column by adopting the wedge-shaped computational domain. At the same time, it allows the flow to pass through the central axis by incorporating the 2D Cartesian flow assumption in the central region. The proposed three-dimensional computational domain can be discretized and solved in Cartesian coordinates with appropriate boundary conditions. When a single layer of cell is used to discretize the computational domain, the simulation becomes essentially two-dimensional although the variation in cell volume has to be accounted for in the computation. It avoids the singularity issue in solving the governing equations over a 2D domain in the cylindrical coordinates. A small section of plate to connect two wedges is recommended to maintain the geometrical similarity between the simulated domain and the cylinder fluidized bed. The cross-section of the small plate can be slightly adjusted to smooth the transition. Here, a flat plate is shown for simplicity. The simulation becomes essentially 
two-dimensional when a single layer of computational cells are used to discretize the cross-section shown in Figure 2.

Figure 2.

The proposed model has been implemented into the open-source code, Multiphase Flow with Interphase eXchanges (MFIX), developed at the National Energy Technology Laboratory (NETL). In MFIX, the multi-fluid, Eulerian-Eulerian approach is used, with each phase treated as an interpenetrating continuum. Mass and momentum conservation equations are solved for the gas and solids (particulate) phases, with appropriate closure relations (Syamlal et al. 1993; Benyahia et al. 2012). Constitutive relations derived from granular kinetic theory are used for the solids phase as summarized in Li et al. 2012. More information on MFIX, as well as detailed documentation on the model equations and the numerical implementation, can be found at the MFIX website, https://mfix.netl.doe.gov. In the current implementation, the 3D computational domain is discretized with only one layer of computational cell in the angular direction as shown in Figure 3 . In the height and radial directions, the simple 2D Cartesian staggered grid is used with scalar quantities solved on the cell centers while the velocities are computed on the cell faces. The velocity component in the angular direction is ignored as the flow is assumed two-dimensional and only one cell in that direction is used. However, the variation in cell size has been accounted for in solving the mass and momentum conservation equations to impose the geometric similarity to the simulated cylindrical column. Detailed numerical implementation on the staggered grid approach in MFIX can be found in Syamlal (1998).

Figure 3. 


\section{Simulation Setup}

The experimental system reported by Reuge et al. (2008) is simulated to test the new method. The system consists of a cylindrical fluidized bed $100 \mathrm{~cm}$ high and $5 \mathrm{~cm}$ in internal diameter operated under ambient conditions. Experiments of two alumina powders of different sizes were reported. Reuge et al. (2008) conducted various numerical simulations of this system mainly for predicting the bed expansion. Their numerical study reported 2D Cartesian and axisymmetric simulations and 3D simulations using cylindrical coordinates that covered several operating conditions for both types of particles. Their study indicated that 3D simulations are necessary for correctly reproducing the experimental bed expansions and fluctuation of bed height, while the 2D simulations widely overestimated both quantities. The 2D Cartesian calculations showed better agreement with the experiments and the 3D simulation than the $2 \mathrm{D}$ axisymmetric calculations, but they still significantly overestimated the bed expansions and fluctuation of bed height.

In the current study, a powder with a mean diameter of $221 \mu \mathrm{m}$ was simulated. Summary of physical properties and numerical parameters used in the simulations are provided in Table 1. The Syamlal-O'Brien (2003) drag correlation was used in this study and was calibrated based on the physical properties of the carrier gas and powder properties to obtain the experimentally measured minimum fluidization velocity by Reuge et al. (2008). Several 2D simulations with the current model and traditional axisymmetric and Cartesian 2D flow assumptions are conducted. In addition, 3D simulations of the cylindrical bed were conducted in Cartesian coordinates with the aid of a cut-cell technique (Dietiker 2013). The second-order Superbee scheme was used for spatial discretization, and the first-order Euler scheme with variable time-step was used for temporal discretization. In all simulations, all physical and numerical parameters are unchanged unless explicitly mentioned.

Table 1.

At the top boundary, a constant pressure is assumed, and particles are allowed to leave the system. For the bottom distributor, a uniform gas velocity is specified, with no solids entering the domain. For the lateral side walls, a non-slip boundary condition for the gas and particle phases is used. The non-slip boundary conditions for the solid phase are used to facilitate comparison between different simulations. Ideally, a partial-slip boundary condition is believed to be more physical for gas-solid fluidized bed simulations. The system is initialized with a packed bed status. Unsteady flow 
simulations are conducted with the transient results saved at a frequency of $50 \mathrm{~Hz}$ for post-processing and analysis.

\section{Results and discussion}

\subsection{Grid Refinement Study}

Ensuring that the CFD results are independent of the grid size prior to meaningful validation and prediction is critical. However, evaluating the grid convergence for the unsteady multiphase flow simulations is difficult (Gel et al. 2013; Li et al. 2014a). In the current study, systematic grid-refinement was conducted for both the 2D and 3D simulations, and the mean voidage and solids velocity are examined to verify the grid convergence. An objective judgment is used to justify the grid convergence by comparing results obtained at different grid resolutions.

The grid-refinement study was first conducted for the 2D Cartesian simulations with a computational domain of $5 \mathrm{~cm} \times 100 \mathrm{~cm}$ using grid resolutions of $25 \times 400,32 \times 512,40 \times 640$, and $50 \times 800$ with a refinement ratio of 1.25 . The simulations are conducted for $100 \mathrm{~s}$ with the last 80 s averaged for analysis. The profiles of cross-sectional average gas holdup along the bed height predicted by different grid resolutions are compared in Figure 4. Overall, all profiles are consistent, and only minor differences can be observed in the upper region of the bed. Reuge et al. (2008) conducted a grid study for the same system. According to their study, a grid resolution of 30X300 for the computational domain of $5 \mathrm{~cm} \times 60 \mathrm{~cm}$ (grid resolution close to $32 \times 512$ in the current study) seemed sufficient to obtain bed expansion and fluctuation of bed height that are independent of the grid resolution. The current observation is consistent with their findings.

Figure 4.

Depending on the quantity of interest and the allowable tolerance, grid convergence can be achieved by different grid resolutions (Li et al. 2014a). Here, we only focus on the mean flow hydrodynamics inside the system, further examination has been made for the radial profiles of mean voidage and vertical solids velocity at the height of $15 \mathrm{~cm}$ above the distributor. As shown in Figure 5, both voidage and vertical solids velocity are affected by the grid resolution. The solids velocity shows more sensitivity to the grid resolution than the voidage. The results in Figure 5 tend to converge at the grid 
resolutions of $40 \times 640$ and 50X800, in which the grid sizes correspond to around 5 or 6 times the particle diameter, which is in agreement with previous grid-resolution studies in the literature (e.g. Wang et al. 2009).

Figure 5.

The contour plots of the mean flow field with respect to voidage and solids vertical velocity are further compared in Figure 6 for different grid resolutions to justify the grid convergence. Converging flow behaviors can be observed in the figure as the grid resolution is increased from left to right. Overall the numerical results show good convergence for the grid resolutions of 40X640 and 50X800. However, certain discrepancies in the solids velocity distribution still exist, although the convergent behavior can be observed for most flow patterns such as bubble paths at the core of the bed and solids backflow near the walls. In addition, the slightly asymmetric flow behavior in the lower region of the bed indicates that the time-averaging might not be sufficiently long. This fact has been confirmed for the grid resolutions of 40X640 and $50 \times 800$ by extending the simulation run to 450 s, which results in a more symmetrical flow field. Comparison between results of 100 s and 450 s simulation runs (results not shown) suggested that 100 s simulation is sufficient to quantitatively capture the major flow hydrodynamic behavior. Therefore, the shorter simulation time (100 sec) is used in this study to investigate the flow hydrodynamics and to compare against the 3D simulations.

Figure 6.

For the 3D simulation, a Cartesian grid is used to discretize the computational domain while the boundary cells are truncated to conform to the boundary surface (Dietiker 2013). This approach has been successfully applied to gas-solids flow with complex geometries (Li et al. 2011, 2012; Dietiker et al. 2013). According to the 2D simulations and experimental measurement, the expended bed height is about $30 \mathrm{~cm}$. To reduce the computational cost of 3D simulations, the height of the computational domain was reduced from $100 \mathrm{~cm}$ to $50 \mathrm{~cm}$. The domain height of $50 \mathrm{~cm}$ has been confirmed as sufficient for the current operating conditions by comparing 3D simulations with different domain heights (results not shown). Four grid resolutions of 25X200X25, $32 \times 256 \times 32,40 \times 320 \times 40$, and $50 \times 400 \times 50$, which are equivalent to the grid resolutions used in the 2D grid study, are used. Similar to the 2D simulations, time averaged results of the last 80 s of 100 s simulation are analyzed. 
Figure 7 presents the radial profiles of mean voidage and vertical solids velocity at the height of $15 \mathrm{~cm}$ predicted by 3D simulations with different grid resolutions. Similar to the $2 \mathrm{D}$ results, the voidage is high in the central region, and solids down flow exists along the walls. The voidage profiles do not show much sensitivity to the grid resolution. However, the solids' vertical velocity profiles exhibit stronger influences by the grid resolution. Considering the small differences between results of 40X320X40 and $50 \times 400 \times 50$, it is reasonable to conclude that the simulation results converge at the grid resolution of $40 \times 320 \times 40$, which is consistent with the conclusion drawn from the 2D grid-refinement study.

Figure 7.

The mean voidage and solids vertical velocity distribution along a central slice plane in the 3D bed are shown in Figure 8 for different grid resolutions. From the voidage distribution, it can be concluded that bed expansion is almost the same for all grids. However, the detailed flow hydrodynamics inside the bed are still sensitive to the grid resolution. As the grid resolution increases, the voidage distribution shows that bubbles formed above the distributor tend to collapse in the central region sooner. This is closely related to the formation of bubbles above the distributor, which is simplified as uniform gas flow conditions. When a finer grid is used, the uniform gas flow tends to become unstable closer to the distributor, and then forms small bubbles. The same conclusion can be drawn from the solids velocity distribution. Overall, the results from the $40 \times 320 \times 40$ and 50X400X50 grids are very close, which indicates a reasonable grid convergence at 40X320X40. Again, slight asymmetric flow behaviors can be observed for both the mean voidage and solids velocity distributions, which suggest that a larger sampling duration is preferable. The required simulation time for the representative mean flow field is closely related to the dominant frequency in the flow behavior. However, due to the inherent multi-scale temporal and spatial variations in the gas-solid multiphase flow, it is difficult to justify a sufficient simulation time for analysis. Because 3D simulations are computationally expensive, only 100s simulations are completed for each run. With extended simulation time, the radial profiles are expected to be smoother and more symmetrical, as demonstrated earlier for the 2D simulation.

Figure 8. 
Based on the above grid study, the grid resolution of 50X800 is used for the $2 \mathrm{D}$ simulation with/without the new method, and the grid resolution of $25 \times 800$ is used for the axisymmetric simulation. For the 3D simulations, the results of fine grid with resolution of $50 \times 400 \times 50$ are presented in the following analysis. To be conservative, the finest grids are used for both 2D and 3D simulations to make sure the numerical results are grid independent.

\subsection{Comparison between various 2D simulations}

Numerical results of different methods are compared in this section to analyze the advantages and disadvantages of each approach. Figure 9 compares the radial profiles by 2D Cartesian simulation, 2D axisymmetric simulation, 2.5D Cartesian simulation with the new computational domain, and 3D simulation. In the figure, the 2D axisymmetric results have been flipped and shifted to facilitate direct comparison. Among all results, the axisymmetric simulation predicts the most distinct flow behavior with the highest solids concentration along the central axis. This has been discussed extensively in the literature and is attributed to the central axis behaving like a free slip wall that confines the solids movement. The axisymmetric simulation predicts a negative mean solids velocity throughout the cross-section. This is again attributed to the central axis that divides the bed and promotes the formation of long slugs as revealed by the simulation results. The presence of long slugs leads to negative mean solids velocity when the slugs rise slowly with particles raining down from the top. The other simulation approaches predict qualitatively consistent results of relatively dilute upflow in the central region and dense downward flow along the wall. However, considerable quantitative differences lie among them.

The numerical results from the 3D simulation are used as reference because no experimental data is available for model validation. Any assumption meant to reduce the true nature of a 3D problem should be compared to the results of a 3D simulation, which is considered in this study as the most accurate. The 3D simulation predicts a denser central region and weaker solids down flow along the wall than in the 2D Cartesian simulations. Compared to the conventional 2D Cartesian simulation, the new 2.5D model yields similar solids distribution but different solids velocity profile. The 2.5D model predicts slightly higher upward solids velocity in the center and much lower downward solids velocity along the wall. For the results of the 2.5D model shown in the figure, the ratio between the half-width plate and column diameter $L / R=0.28$ is used. $A$ parametric study of this parameter has been conducted and is presented in the next section. Overall, the $2.5 \mathrm{D}$ model improved solids velocity predictions close to the wall compared to the 3D results because it considered the geometry of the cylindrical column and its wall effect correctly. 
Figure 9.

\subsection{Parametric Study}

In the current model, the ratio between the plate half width, $L$, and the wedge radius (or bed radius), $R$, is an important parameter determining the transition from 2D Cartesian assumption to axisymmetric flow assumption. Unfortunately, the literature provides no guidance that can be used to determine this parameter. Generally speaking, this ratio should be small to eliminate the geometrical discrepancy caused by the 2D flow Cartesian assumption. On the other hand, it has to be large enough to allow particles passing the central axis and overcome the unphysical solids accumulation in the central region. The introduction of the plate region leads to a geometric discrepancy in the cross-section compared to the ideally axisymmetric domain. This discrepancy can be estimated as

$$
\phi=\frac{1}{2}(L / R)^{2}
$$

For the operating condition listed in Table 1 , different values of $L / R(0.04,0.12,0.20$, $0.28,0.36$, and 0.44 ) were tested.

Figure 10 shows the radial profiles at the height of $15 \mathrm{~cm}$ predicted by $2.5 \mathrm{D}$ simulation with different $L / R$ values. As can be seen in the figure, when the ratio is small, the results are close to those of axisymmetric simulations with high solids concentration in the central region. As a consequence, the small L/R prevents the vigorous solids exchange between two wedge sections. Because of the limited solids exchange, the simulation tends to predict asymmetric flow behavior as shown in the figure. The asymmetric flow behavior can be overcome by extending the simulation time or promoting solids exchange between two sections. As the ratio between $L$ and $R$ increases, the simulation results become similar to the 2D Cartesian results with stronger solids down flow along the walls and demonstrate more symmetric flow profiles. For the current problem, L/R=0.28 and 0.36 seem to present a proper compromise between axisymmetric and 2D flow assumptions. 
Figure 10.

Figure 11 further compares the mean flow fields predicted with different $L / R$ values. As evident from the figure, for low L/R values, the flow exhibits strong asymmetry due to the limited radial flow exchange between two wedges connected by the thin plate. In addition, the transition from wedge to the 2D plane leads to slight discontinuity in the voidage distribution for low values of $L / R$. This discontinuity becomes less pronounced as this ratio is increased.

Figure 11.

In the new 2.5D model, another parameter is the angle $\alpha$ of the wedge as shown in Figure 2. A sensitivity analysis is undertaken here to investigate the effect of this parameter on flow profiles.

Figure 12 compares the results predicted by the 2.5D simulations with angles of $1,0.1$, and $0.01 \mathrm{rad}$ for $L / R=0.36$. Clearly, the results are consistent, and no significant effect from the angle is observed. This observation also verifies the implementation of the new model.

Figure 12.

\subsection{Further evaluation}

Two cases with higher superficial gas velocities of 0.16 and $0.25 \mathrm{~m} / \mathrm{s}$ are simulated to further evaluate the performance of the new model. For higher superficial gas velocities, the full system of $1 \mathrm{~m}$ height is simulated to avoid solids escaping due to a low domain height. For the 3D simulations, the grid resolution of $40 \times 640 \times 40$ is used according to the grid study reported previously. For the 2D simulations, the grid resolution of 50X800 is still used. No axisymmetric flow simulation is conducted for high superficial gas velocities. Both $2 \mathrm{D}$ and $2.5 \mathrm{D}$ simulation results are compared against the $3 \mathrm{D}$ results as shown in Figure 13 and Figure 14. It can be seen that both 2D and 2.5D simulations consistently over-predicted the solids concentration in the central region of the bed for two operating conditions. The differences are mainly presented in the solids velocity profiles. The 2D Cartesian simulations consistently over-predict the maximum downward velocity near the wall by around $100 \%$ compared to the 3D simulations for 
both conditions. The $2.5 \mathrm{D}$ model predicts a better solids velocity profile in the wall region but over-predicts the solids velocity in the center region, especially for the case with a superficial gas velocity of $0.25 \mathrm{~m} / \mathrm{s}$. Overall, the new model results compare more favorably to the $3 \mathrm{D}$ results than to the traditional $2 \mathrm{D}$ simulation. The radial profiles for both conditions present better symmetry compared to the case with low superficial gas velocity. For high superficial gas velocities, much stronger solids circulations exist inside the system for which the simulation time of 100s becomes sufficient to achieve more symmetrical mean flow fields.

Figure 13.

Figure 14.

Figure 15 summarizes the comparison of mean flow fields predicted by different approaches for all three operating conditions simulated. The axisymmetric simulations are not included because they are far different from the rest and believed to be unphysical. The bed expansions predicted by the three models are slightly different. The 3D simulation predicts the least bed expansion. With the same computational cost between the $2 \mathrm{D}$ and $2.5 \mathrm{D}$ simulations, the $2.5 \mathrm{D}$ model which accounts for the cylindrical geometry yields slightly better agreement with the $3 \mathrm{D}$ results as far as the bed expansion is concerned. When the mean voidage and solids velocity are compared, there still exist significant discrepancies among different simulations though they all predict qualitatively similar flow patterns. Overall, the $2.5 \mathrm{D}$ model seems to demonstrate better consistency with the 3D results. The strong discrepancy between 2D and 3D simulations are believed to be related to the inherent 3D nature of the gas-solids flow that the 2D simulations fail to account for. The current test problem is a small bubbling fluidized bed in which slugging tends to take place. Hence the applicability of the proposed model requires more tests covering a wider range of system configuration and operating conditions. However, we believe that this new model should provide improved quantitative prediction as far as the 2D simulation is concerned. Of course, the 2D flow assumption has inherent limitations on predicting the complex gas-solids flow in a real system, so we suggest conducting 3D simulations whenever possible.

Figure 15. 


\section{Conclusion}

In this study, a new 2.5D model is proposed for simulating cylindrical gas-solids bubbling fluidized beds by combining 2D axisymmetric and 2D Cartesian flow assumptions. The current method attempts to overcome the singularity issue associated with the axisymmetric assumption and improves the 2D Cartesian flow assumption by incorporating the correct geometric information in the simulation. This new method has been tested for a small bubbling fluidized bed system for which various 2D and 3D simulations have been conducted. Detailed comparison of the flow hydrodynamics are made after careful grid studies to ensure the results are grid independent. Through comparison among results obtained with different modeling approaches, the new 2.5D model produces improved results compared to the 2D Cartesian and 2D axisymmetric assumptions. Certain discrepancies exist between the 2D and 3D simulation results that are believed to be inherent limitations of the 2D assumption. Full 3D simulations are still preferred to account for the complex geometry and inherent 3D flow behavior of a real system.

\section{Acknowledgements}

This technical effort was performed in support of the U.S. Department of Energy, Office of Fossil Energy's Carbon Capture Simulation Initiative (CCSI) through the National Energy Technology Laboratory under the RES contract DE-FE0004000.

\section{Disclaimer}

This report was prepared as an account of work sponsored by an agency of the United States Government. Neither the United States Government nor any agency thereof, nor any of their employees, makes any warranty, express or implied, or assumes any legal liability or responsibility for the accuracy, completeness, or usefulness of any information, apparatus, product, or process disclosed, or represents that its use would not infringe privately owned rights. Reference herein to any specific commercial product, process, or service by trade name, trademark, manufacturer, or otherwise does not necessarily constitute or imply its endorsement, recommendation, or favoring by the United States Government or any agency thereof. The views and opinions of authors expressed herein do not necessarily state or reflect those of the United States Government or any agency thereof. 


\section{Reference}

Benyahia, S., Syamlal, M., O'Brien, T.J. 2012. Summary of MFIX Equations.

https://mfix.netl.doe.gov/documentation/MFIXEquations2012-1.pdf.

Cabezas-Gomez, L., Milioli, F.E., 2003. Numerical study on the influence of various physical parameters over the gas-solid two-phase flow in the 2D riser of a circulating fluidized bed. Powder Technology 132, 216-225.

Cammarata, L., Lettieri, P., Micale, G.D.M., Colman, D., 2003, 2D and 3D CFD simulations of bubbling fluidized beds using Eulerian-Eulerian models. International Journal of Chemical Reactor Engineering A48, 1.

Cloete, S., Johansen,S.T., Amini,S., 2013. Investigation into the effect of simulating a 3D cylindrical fluidized bed reactor on a 2D plane. PowderTechnology 239, 21-35.

Dietiker, J.F., 2013. Multiphase Flow with Interphase eXchanges Cartesian Grid User Guide, from https://mfix.netl.doe.gov/documentation/Cartesian_grid_user_guide.pdf, National Energy Technology Laboratory.

Dietiker, J.F., Li, T., Garg, R., Shahnam, M., 2013. Cartesian grid simulations of gas-solids flow systems with complex geometry, Powder Technology 235, 696-705.

Drake, J.B, Heindel, T.J., 2011. The repeatability and uniformity of 3D fluidized beds, Powder Technology 213, 148-154.

Du, B., Warsito, W., Fan, L.S. 2005, ECT studies of gas-solid fluidized beds of different diameters, Industrial \& Engineering Chemistry Research 44, 5020-5030.

Gel, A., Li, T., Shahnam, M., Syamlal, M., 2013. Validation and Uncertainty Quantification of a multiphase flow CFD model, Industrial \& Engineering Chemistry Research 52, 11424-11435.

Li, T., Zhang, Y., Grace, J.R., Bi, X., 2010, Numerical investigation of gas mixing in gassolid fluidized beds, AIChE Journal 56, 2280-2296.

Li, T., Dietiker, J.F., Zhang, Y., Shahnam, M., 2011. Cartesian grid simulations of bubbling fluidized bed with a horizontal tube bundle, Chemical Engineering Science 8, 6220-6231.

Li, T., Dietiker, J.F., Shahnam, M., 2012. MFIX simulation of NETL/PSRI Challenge Problem of circulating fluidized bed, Chemical Engineering Science 84, 746-760.

Li, T., Gel, A., Pannala, S., Shahnam, M., Syamlal, M., 2014a. CFD simulations of circulating fluidized bed risers, Part I: grid study, Powder Technology 254, 170-180. 
Li, T., Pannala, S., Shahnam, M., 2014b. CFD simulations of circulating fluidized bed risers, Part II: evaluation of differences between 2D and 3D simulations, Powder Technology 254, 115-124.

Lindborg, H., Lysberg, M., Jakobsen, H.A., 2007. Practical validation of the two-fluid model applied to dense gas-solid flows in fluidized beds, Chemical Engineering Science 62, 5854-5869.

Malcus, S., Chaplin, G., Pugsley T., 2010. The hydrodynamics of the high-density bottom zone in a CFB riser analyzed by means of electrical capacitance tomography (ECT), Chemical Engineering Science 55, 4129-4138.

Pain, C.C., Mansoorzadeh, S., de Oliveira, C.R.E., 2001. A study of bubbling and slugging fluidised beds using the two-fluid granular temperature model, International Journal of Multiphase Flow 27, 527-551.

Pain, C.C., Mansoorzadeh, S., Gomes, J.L.M., de Oliveira, C.R.E., 2002. A numerical investigation of bubbling gas-solid fluidized bed dynamics in 2-D geometries, Powder Technology $128,56-77$.

Peirano, E., Delloume, V., Leckner, B., 2001, Two- or three-dimensional simulations of turbulent gas-solid flows applied to fluidization. Chemical Engineering Science. 56 47874799.

Reuge, N., Cadoret, L., Coufort-Saudejaud, C., Pannala, S., Syamlal, M., Caussat, B., 2008. Multifluid Eulerian modeling of dense gas-solids fluidized bed hydrodynamics: Influence of the dissipation parameters, Chemical Engineering Science, 63, 5540-5551.

Sun, B., Gidaspow, D., 1999. Computation of circulating fluidized-bed riser flow for the Fluidization VIII benchmark test, Industrial \& Engineering Chemistry Research 38, 787792.

Syamlal, M., Rogers, W., O'Brien. T.J. 1993. MFIX documentation: Theory guide. Morgantown: U.S. Department of Energy (DOE), Morgantown Energy Technology Center. Syamlal,M., 1998. MFIX documentation: numerical guide, Tech. Rep. DOE/MC313465824, NTIS/DE98002029, National Energy Technology Laboratory, Department of Energy.

Syamlal, M., O'Brien, T.J., 2003.Fluid dynamic simulation of $\mathrm{O} 3$ decomposition in a bubbling fluidized bed, AIChE Journal 49, 2793-2801.

Xie, N., Battaglia, F., Pannala, S., 2008a. Effects of using two- versus three-dimensional computational modeling of fluidized beds - Part I, hydrodynamics. Powder Technology 182, 1-13. 
Xie, N., Battaglia, F., Pannala, S., 2008b. Effects of using two- versus three-dimensional computational modeling of fluidized beds: Part II, budget analysis. Powder Technology $182,14-24$.

Wang, J., van der Hoef, M.A., Kuipers, J.A.M., 2009. Why the two-fluid model fails to predict the bed expansion characteristics of Geldart A particles in gas-fluidized beds: $A$ tentative answer. Chemical Engineering Science 64, 622-625.

Weber, J.M., Mei, J.S., 2013. Bubbling fluidized bed characterization using Electrical Capacitance Volume Tomography (ECVT), Powder Technology 242, 40-50.

Zhang, W., Tung, Y., Johnsson, F., 1991. Radial voidage profiles in fast fluidized beds of different diameters, Chemical Engineering Science 46, 3045-3052. 
Table 1 . Summary of physical properties and numerical parameters used in the simulations.

\begin{tabular}{|l|l|l|l|}
\hline Parameter & Value & Parameter & Value \\
\hline Diameter (cm) & 5 & Height $(\mathrm{cm})$ & 100 \\
\hline $\begin{array}{l}\text { Superficial gas } \\
\text { velocity }(\mathrm{m} / \mathrm{s})\end{array}$ & 0.12 & Bed inventory $(\mathrm{kg})$ & 0.8 \\
\hline Temperature (K) & 297 & Pressure (atm) & 1 \\
\hline Gas viscosity (Pa.s) & $1.8 \mathrm{e}-5$ & $\begin{array}{l}\text { Gas molecular } \\
\text { weight }(\mathrm{kg} / \mathrm{kmol})\end{array}$ & 28.8 \\
\hline $\begin{array}{l}\text { Particle diameter } \\
(\mu \mathrm{m})\end{array}$ & 221 & $\begin{array}{l}\text { Particle density } \\
\left(\mathrm{kg} / \mathrm{m}^{3}\right)\end{array}$ & 3900 \\
\hline $\begin{array}{l}\text { Inter-particle } \\
\text { restitution } \\
\text { coefficient }\end{array}$ & 0.8 & $\begin{array}{l}\text { Particle-wall } \\
\text { restitution } \\
\text { coefficient }\end{array}$ & 0.8 \\
\hline $\begin{array}{l}\text { Angle of inter- } \\
\text { particle friction } \\
\text { (deg) }\end{array}$ & 30 & $\begin{array}{l}\text { Particle-wall } \\
\text { frictional angle } \\
\text { (deg) }\end{array}$ & 30 \\
\hline
\end{tabular}




\section{List of Figures}

Figure 1. Schematic of numerical simulations of (a) 3D cylindrical fluidized bed with (b)

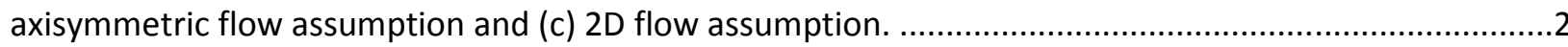

Figure 2. Proposed computational domain for fluidized bed simulations (Top view) ..............................3

Figure 3. Illustration of the computational domain and discretization ................................................4

Figure 4. Axial profiles of average gas holdup predicted by the 2D Cartesian simulations with

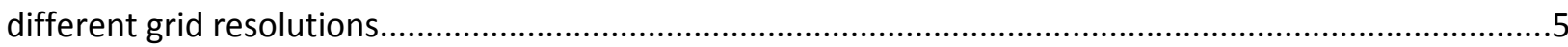

Figure 5. Radial profiles of (a) voidage and (b) vertical solids velocity at the height of $15 \mathrm{~cm}$ predicted by the 2D Cartesian simulations with different grid resolutions.

Figure 6. Mean flow fields predicted by the 2D simulations with different grid resolutions (a) voidage (b) solids vertical velocity (cm/s) (Grid resolutions from left to right: $25 \times 400,32 \times 512$, 40X640, and 50X800).

Figure 7. Radial profiles of (a) voidage and (b) vertical solids velocity at the height of $15 \mathrm{~cm}$ predicted by the 3D simulations with different grid resolutions.

Figure 8. Mean flow fields predicted by the 3D simulations with different grid resolutions (a) voidage (b) solid vertical velocity (cm/s) (Grid resolutions from left to right: 25X200X25, $32 \times 256 \times 32,40 \times 320 \times 40$, and 50X400X50).

Figure 9. Radial profiles of (a) voidage and (b) vertical solids velocity at the height of $15 \mathrm{~cm}$ predicted by different simulations ( $L / R=0.28$ for the $2.5 \mathrm{D}$ model). 10

Figure 10. Radial profiles of (a) voidage and (b) vertical solids velocity at the height of $15 \mathrm{~cm}$ predicted by the $2.5 \mathrm{D}$ simulations with different $\mathrm{L} / \mathrm{R}$ ratios.

Figure 11. Mean flow fields predicted by the 2.5D simulations with different $L / R$ ratios (a) voidage (b) solids vertical velocity ( $\mathrm{cm} / \mathrm{s}$ ) (from left to right: $L / R=0.20,0.28,0.36$, and 0.44 ).

Figure 12. Radial profiles of (a) voidage and (b) vertical solids velocity at the height of $15 \mathrm{~cm}$ predicted by the present model with different wedge angles noted here as $d z$ ( rad) for $L / R=0.36$.

Figure 13. Radial profiles of (a) voidage and (b) vertical solids velocity at the height of $15 \mathrm{~cm}$ predicted by different simulations for the case with superficial gas velocity of $0.16 \mathrm{~m} / \mathrm{s}(\mathrm{L} / \mathrm{R}=0.28$ for the $2.5 \mathrm{D}$ model).

Figure 14. Radial profiles of (a) voidage and (b) vertical solids velocity at the height of $15 \mathrm{~cm}$ predicted by different simulations for the case with superficial gas velocity of $0.25 \mathrm{~m} / \mathrm{s}(\mathrm{L} / \mathrm{R}=0.28$ for the $2.5 \mathrm{D}$ model).

Figure 15. Mean flow fields predicted by different simulations (a) $U g=0.12 \mathrm{~m} / \mathrm{s}$, (b) $U g=0.16 \mathrm{~m} / \mathrm{s}$, (c) $U g=0.25 \mathrm{~m} / \mathrm{s}(\mathrm{L} / \mathrm{R}=0.28$ for the 2.5D model, sub-figures from left to right are $2 \mathrm{D}, 2.5 \mathrm{D}$, and $3 \mathrm{D}$ simulation results). 


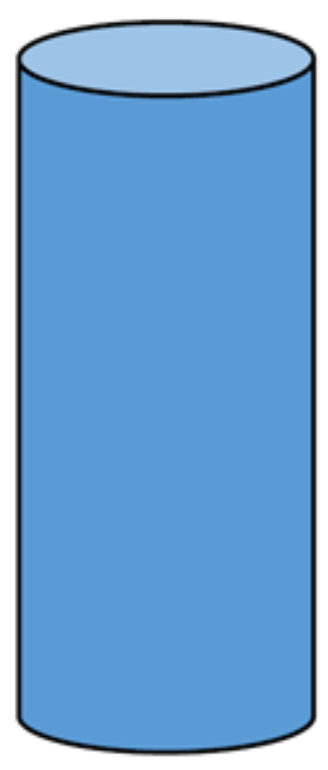

(a)

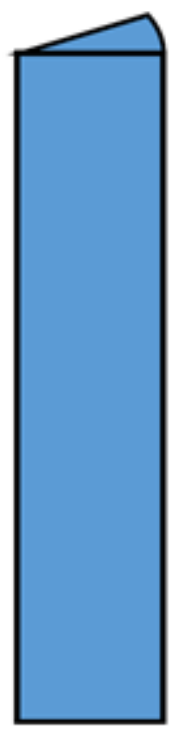

(b)

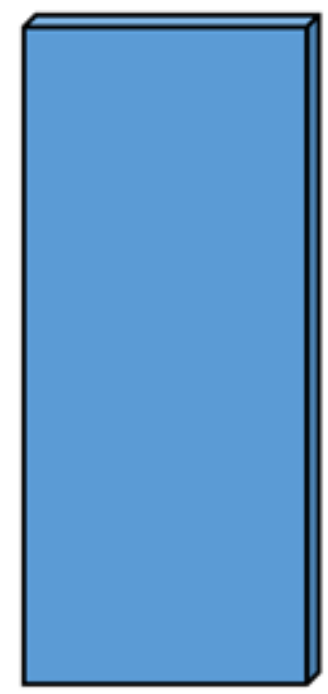

(c)

Figure 1. Schematic of numerical simulations of (a) 3D cylindrical fluidized bed with (b) axisymmetric flow assumption and (c) 2D flow assumption. 


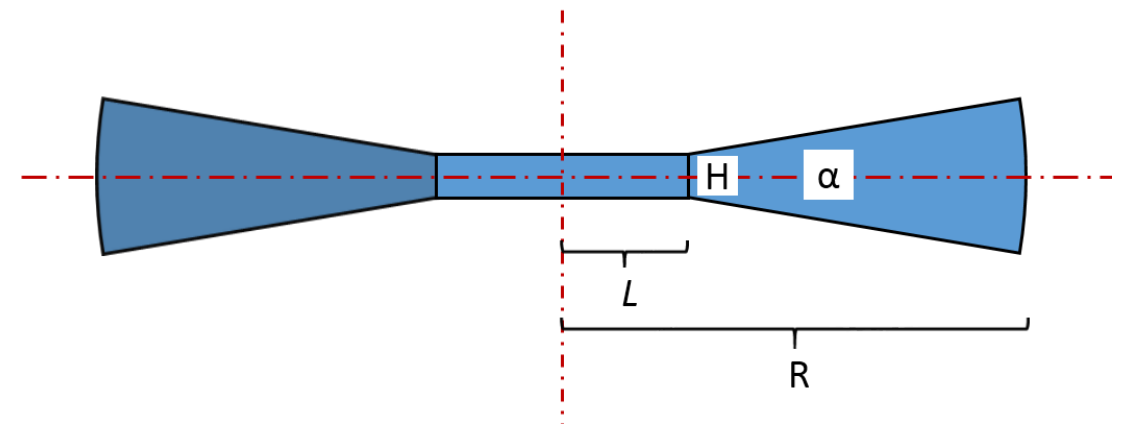

Figure 2. Proposed computational domain for fluidized bed simulations (Top view). 


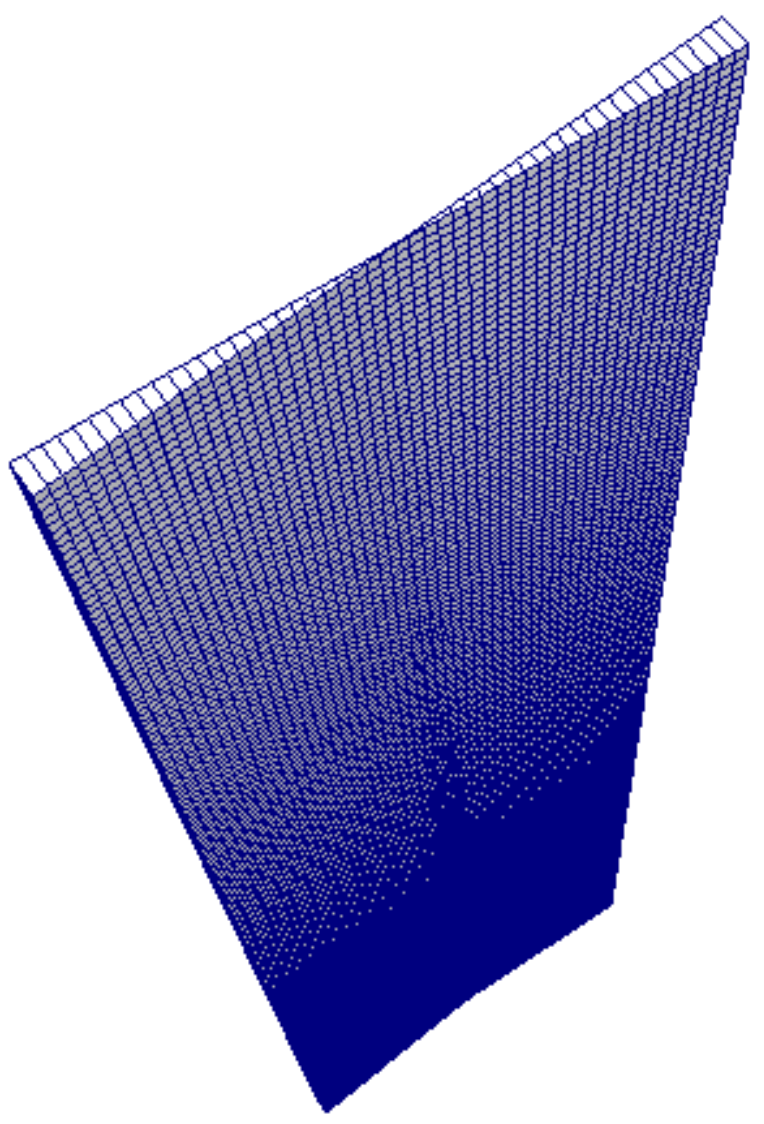

Figure 3. Illustration of the computational domain and discretization 


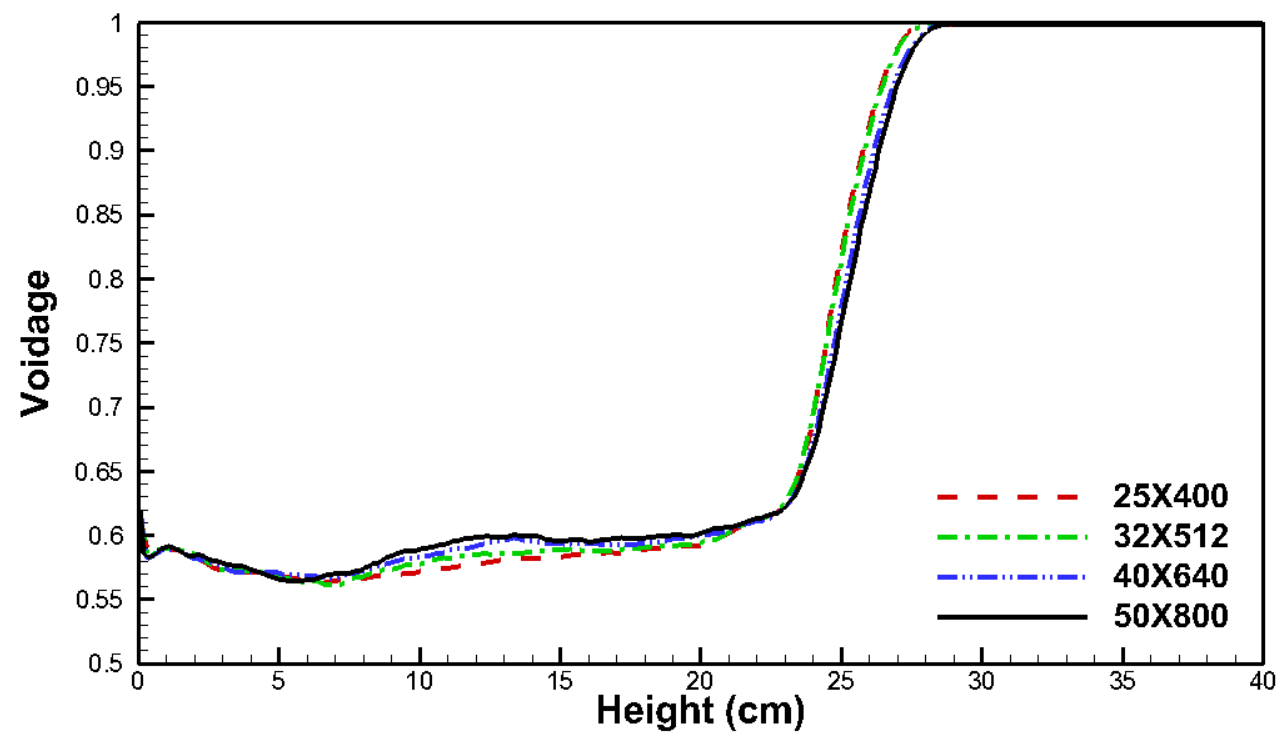

Figure 4. Axial profiles of average gas holdup predicted by the 2D Cartesian simulations with different grid resolutions. 


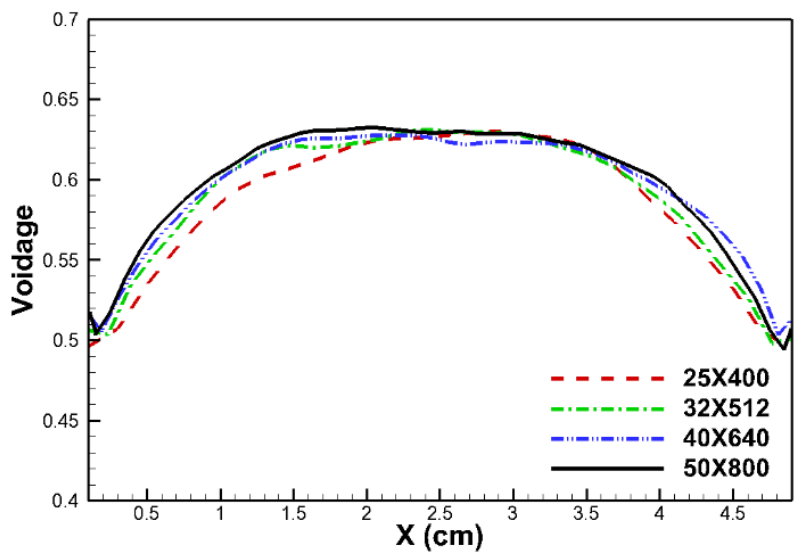

(a)

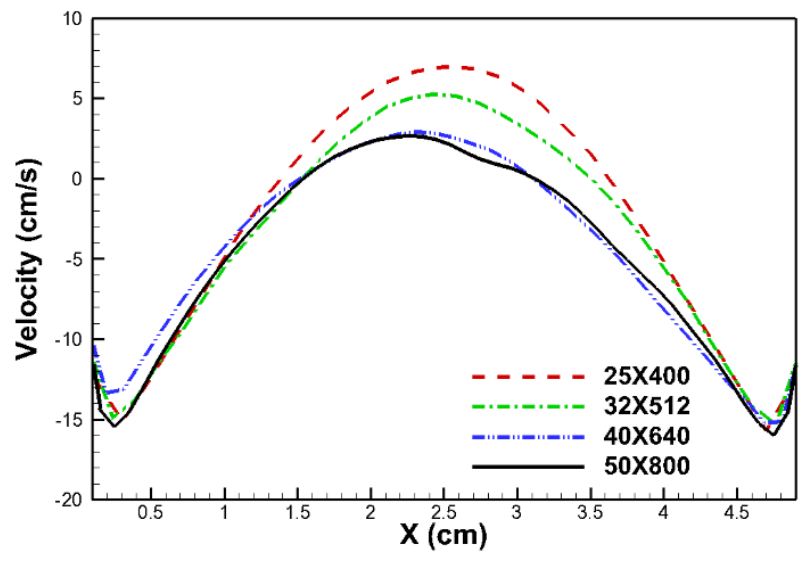

(b)

Figure 5. Radial profiles of (a) voidage and (b) vertical solids velocity at the height of $15 \mathrm{~cm}$ predicted by the 2D Cartesian simulations with different grid resolutions. 

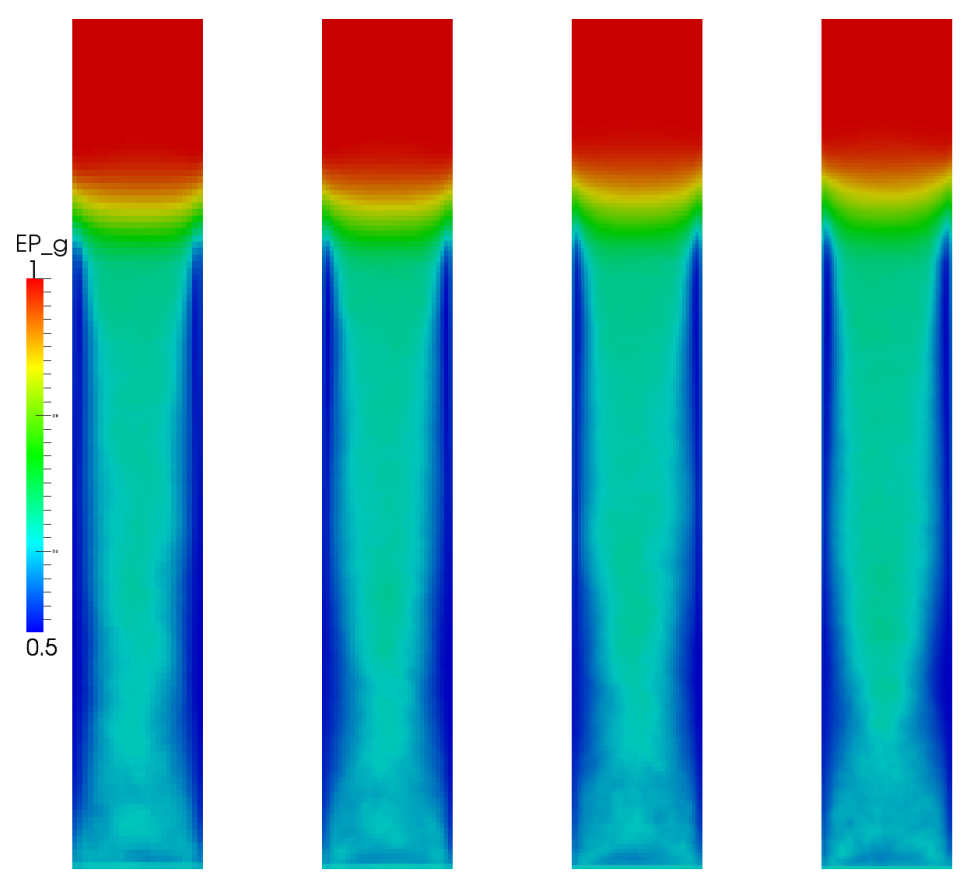

(a)
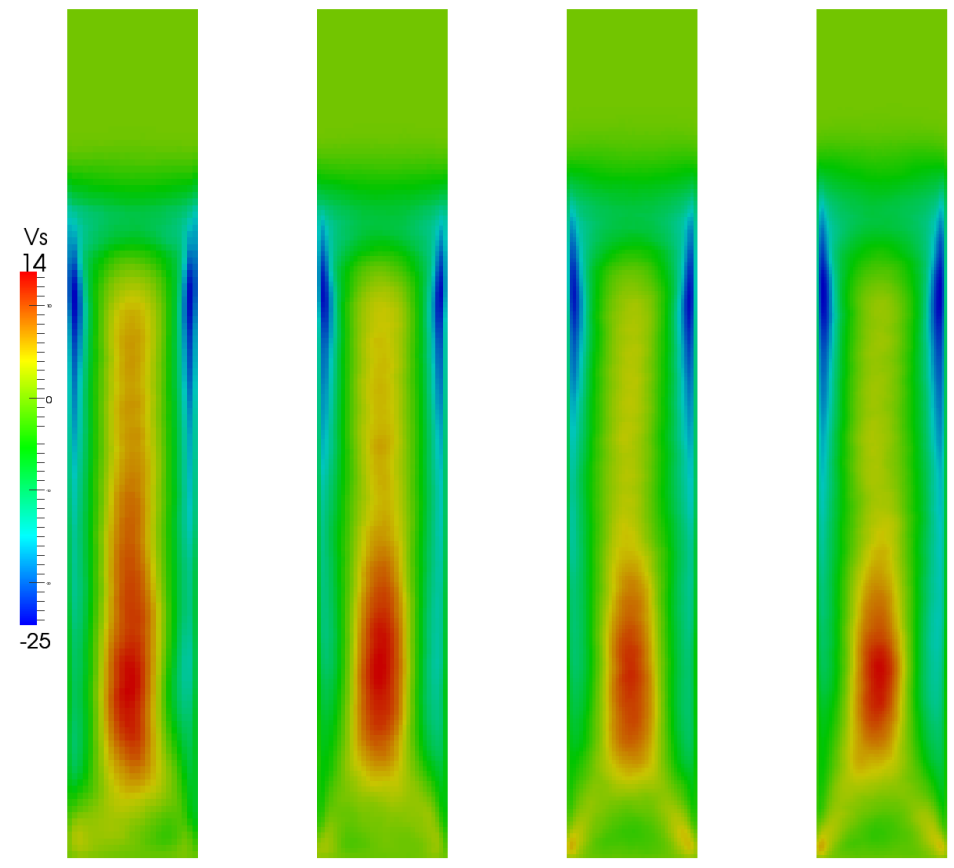

(b)

Figure 6. Mean flow fields predicted by the 2D simulations with different grid resolutions (a) voidage (b) solids vertical velocity $(\mathrm{cm} / \mathrm{s}$ ) (Grid resolutions from left to right: 25X400, 32X512, 40X640, and 50X800). 


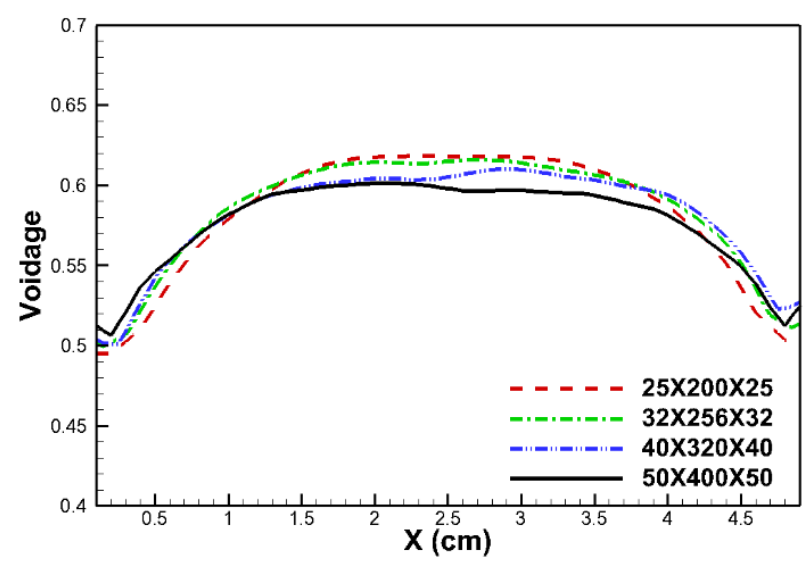

(a)

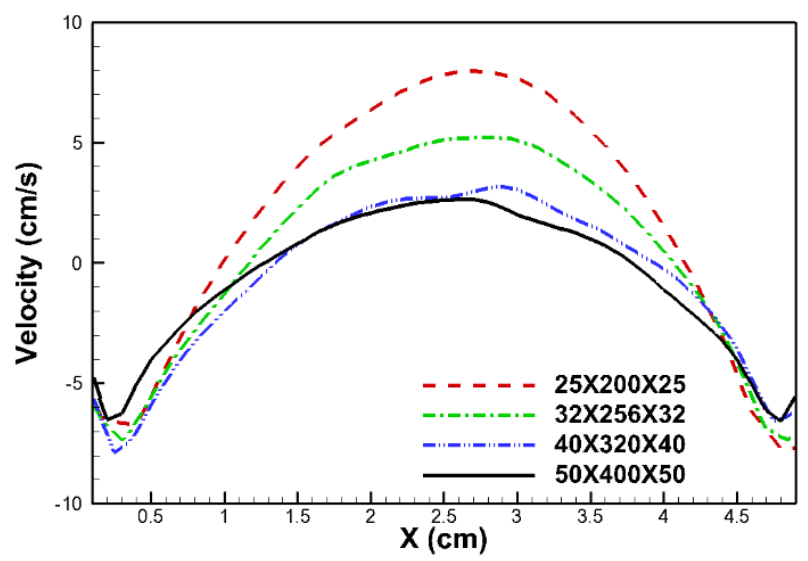

(b)

Figure 7. Radial profiles of (a) voidage and (b) vertical solids velocity at the height of $15 \mathrm{~cm}$ predicted by the 3D simulations with different grid resolutions. 

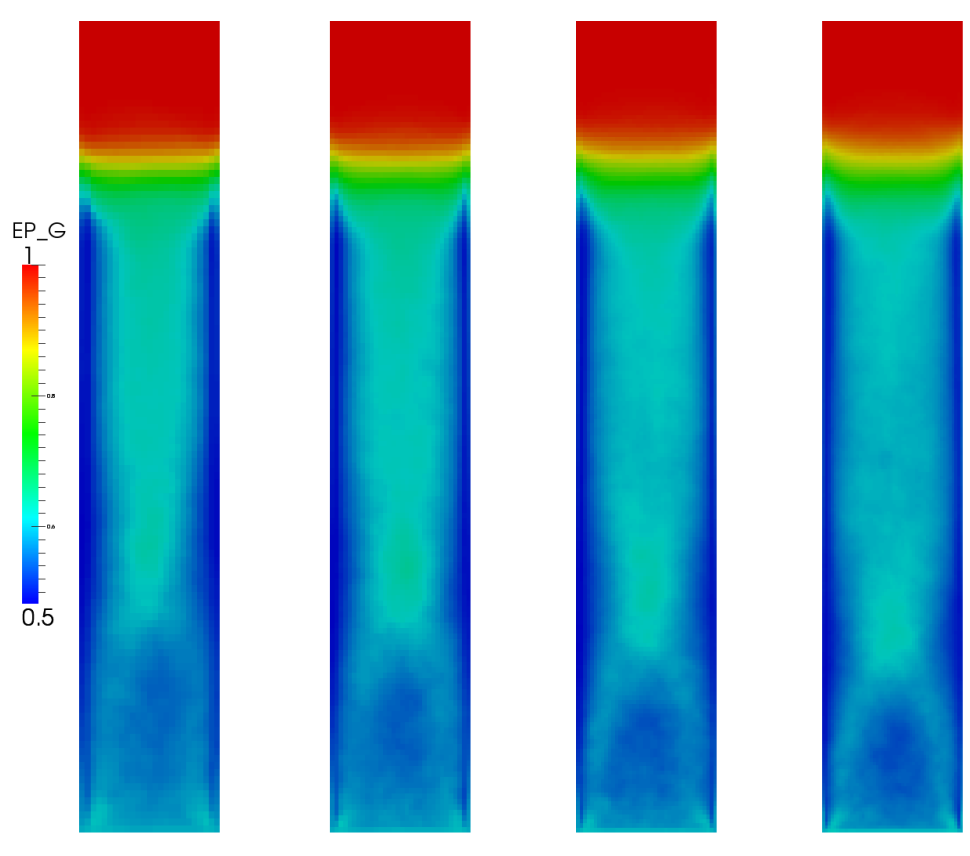

(a)
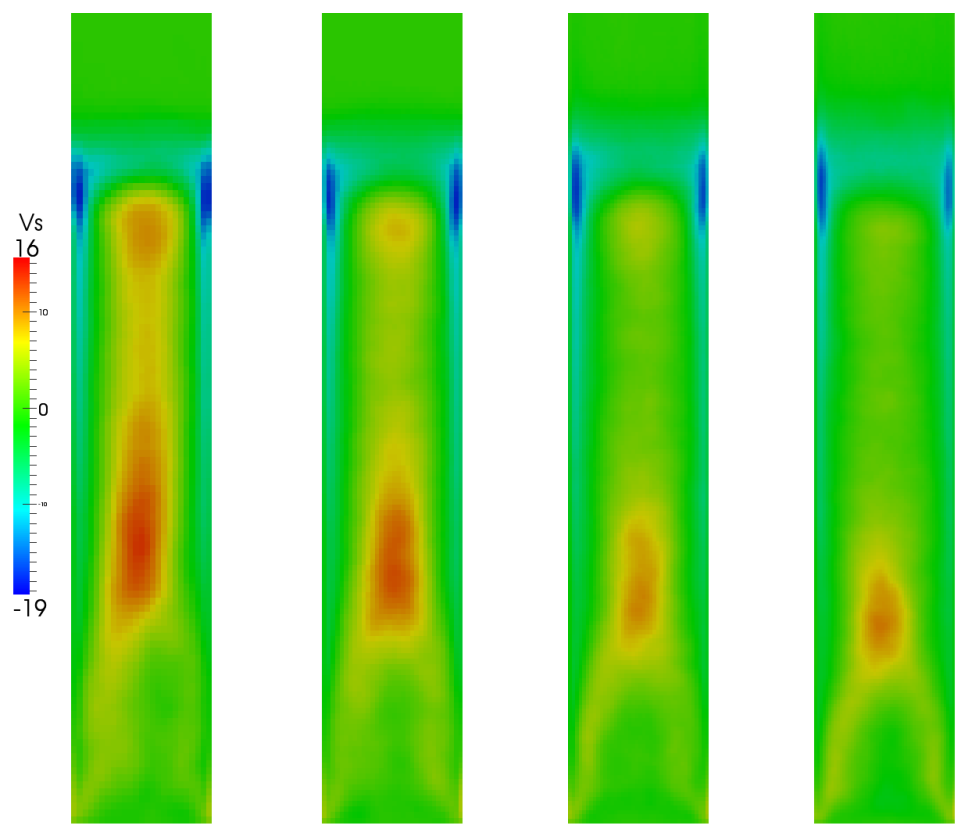

(b)

Figure 8. Mean flow fields predicted by the 3D simulations with different grid resolutions (a) voidage (b) solid vertical velocity $(\mathrm{cm} / \mathrm{s})$ (Grid resolutions from left to right: 25X200X25, 32X256X32, 40X320X40, and 50X400X50). 


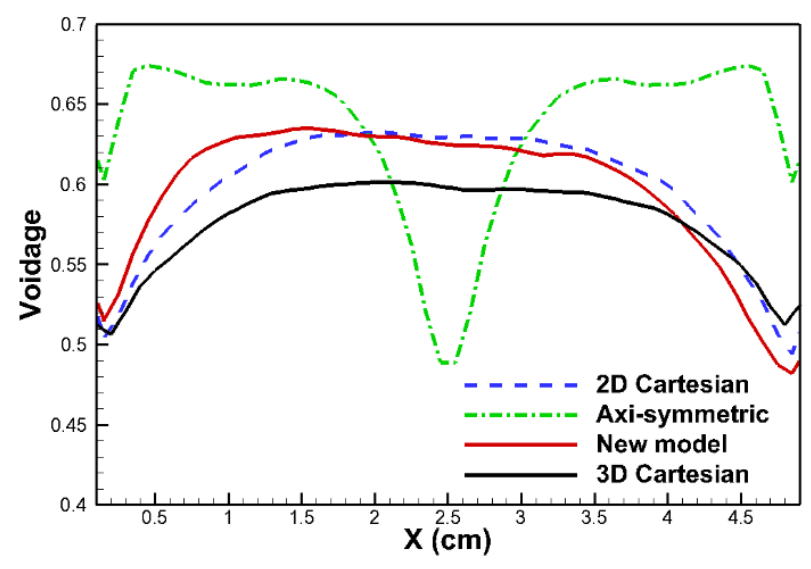

(a)

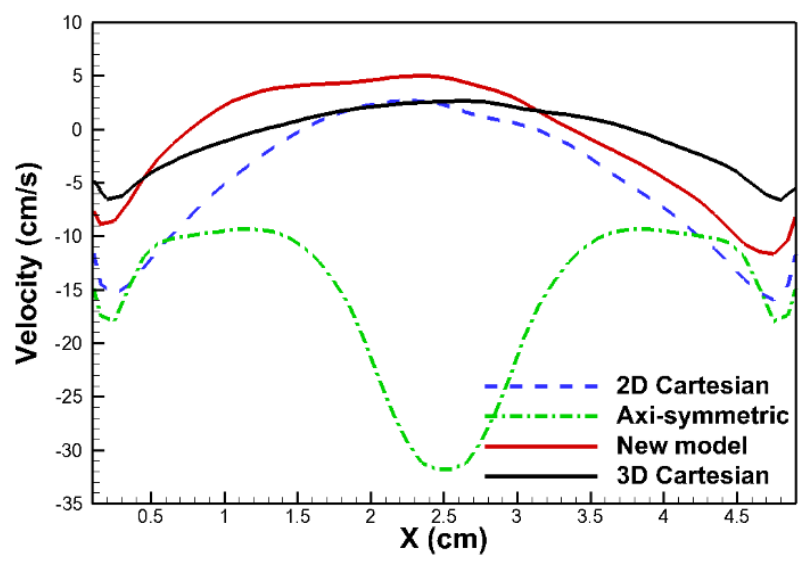

(b)

Figure 9. Radial profiles of (a) voidage and (b) vertical solids velocity at the height of $15 \mathrm{~cm}$ predicted by different simulations ( $L / R=0.28$ for the $2.5 \mathrm{D}$ model). 


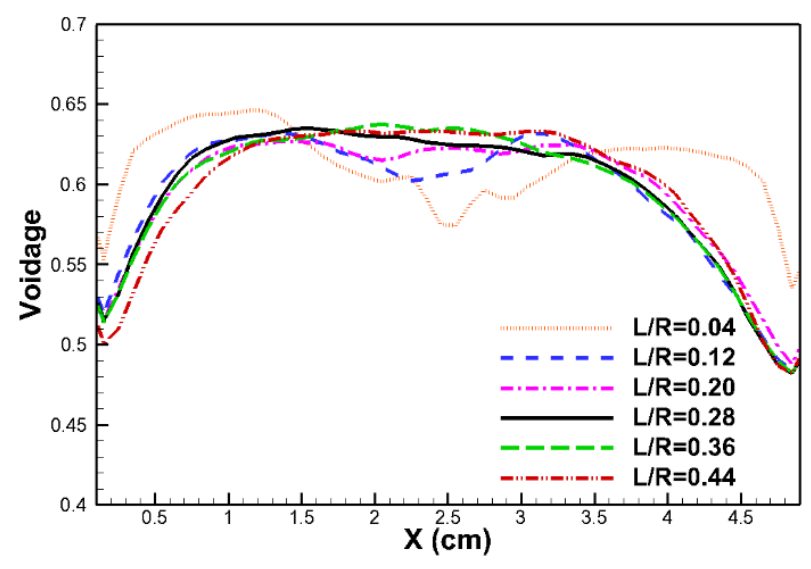

(a)

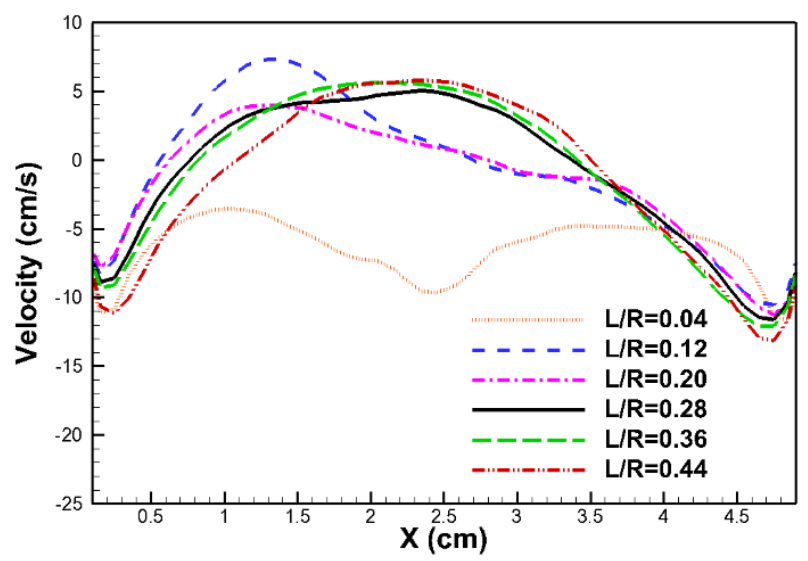

(b)

Figure 10. Radial profiles of (a) voidage and (b) vertical solids velocity at the height of $15 \mathrm{~cm}$ predicted by the $2.5 \mathrm{D}$ simulations with different $L / R$ ratios. 

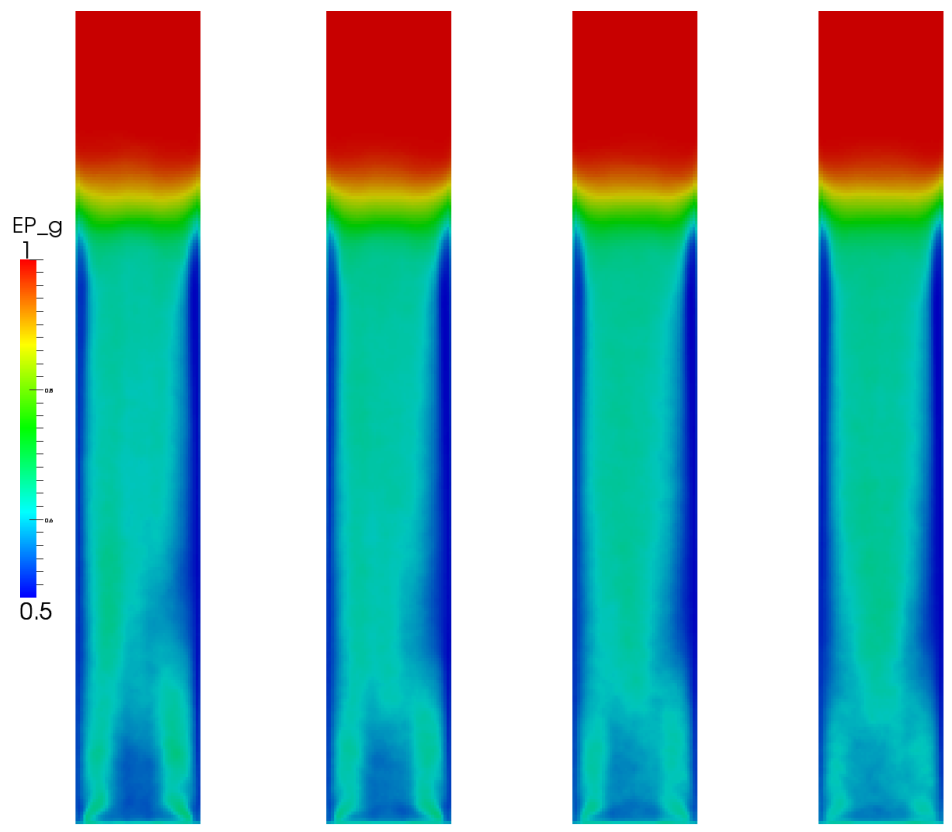

(a)
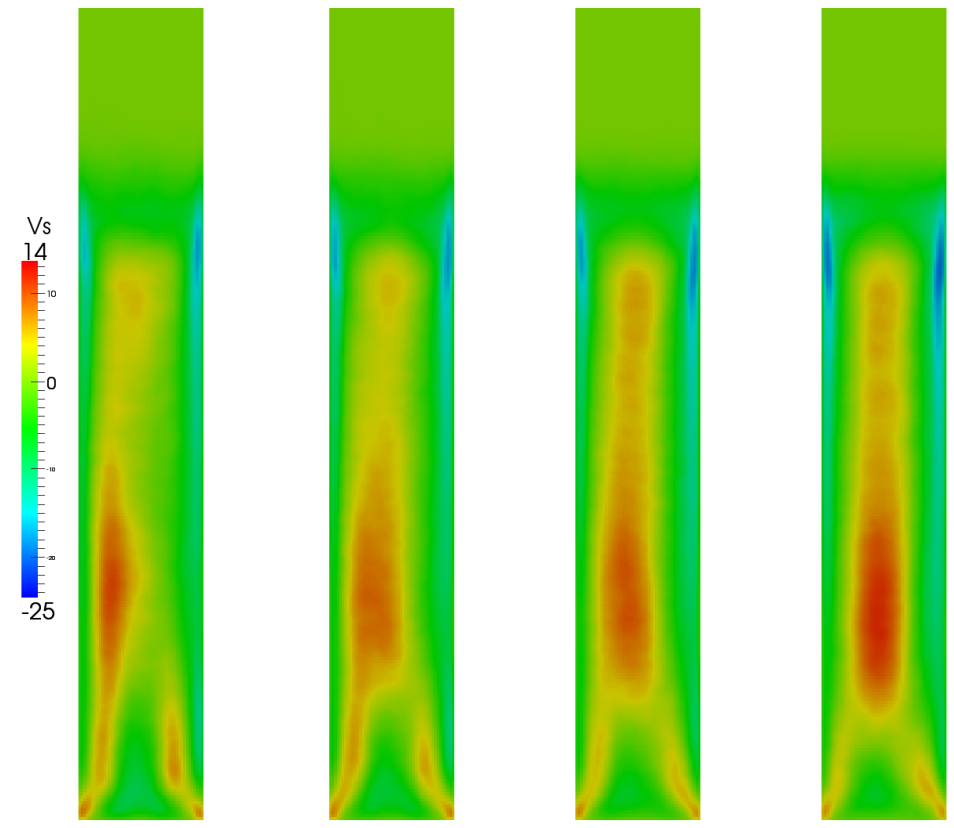

(b)

Figure 11. Mean flow fields predicted by the 2.5D simulations with different $L / R$ ratios (a) voidage $(b)$ solids vertical velocity $(\mathrm{cm} / \mathrm{s}$ ) (from left to right: $L / R=0.20,0.28,0.36$, and 0.44). 


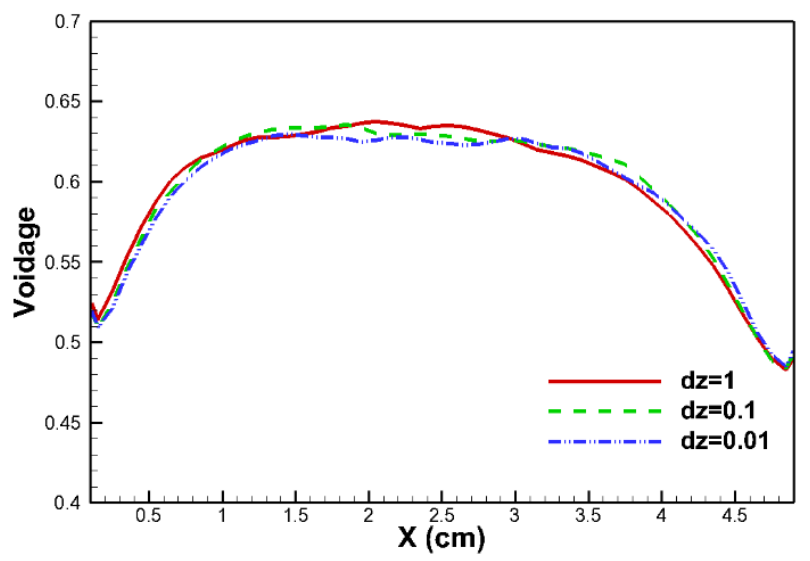

(a)

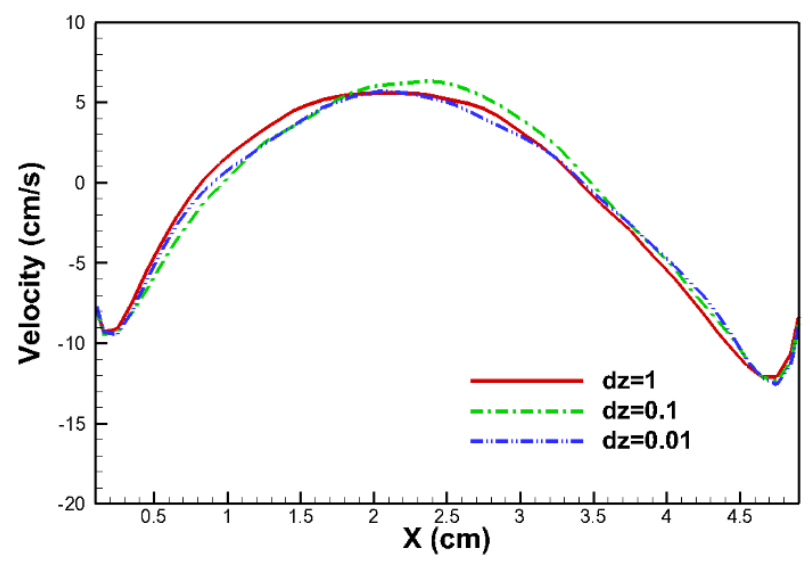

(b)

Figure 12. Radial profiles of (a) voidage and (b) vertical solids velocity at the height of $15 \mathrm{~cm}$ predicted by the present model with different wedge angles noted here as $\mathrm{dz}$ (rad) for $L / R=0.36$. 


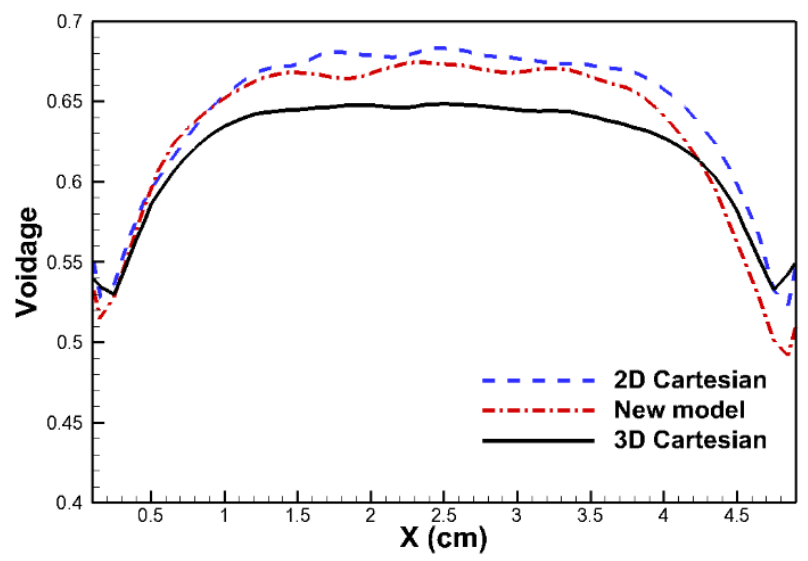

(a)

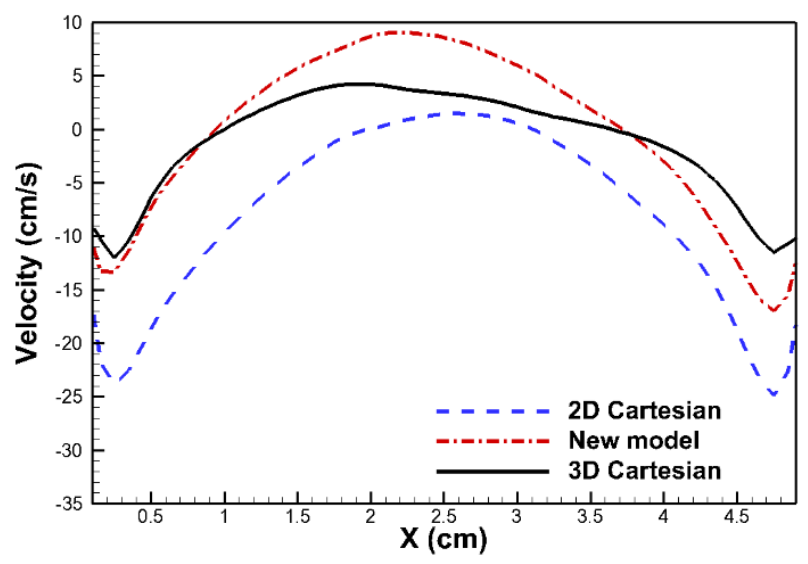

(b)

Figure 13. Radial profiles of (a) voidage and (b) vertical solids velocity at the height of $15 \mathrm{~cm}$ predicted by different simulations for the case with superficial gas velocity of $0.16 \mathrm{~m} / \mathrm{s}(\mathrm{L} / \mathrm{R}=0.28$ for the $2.5 \mathrm{D}$ model). 


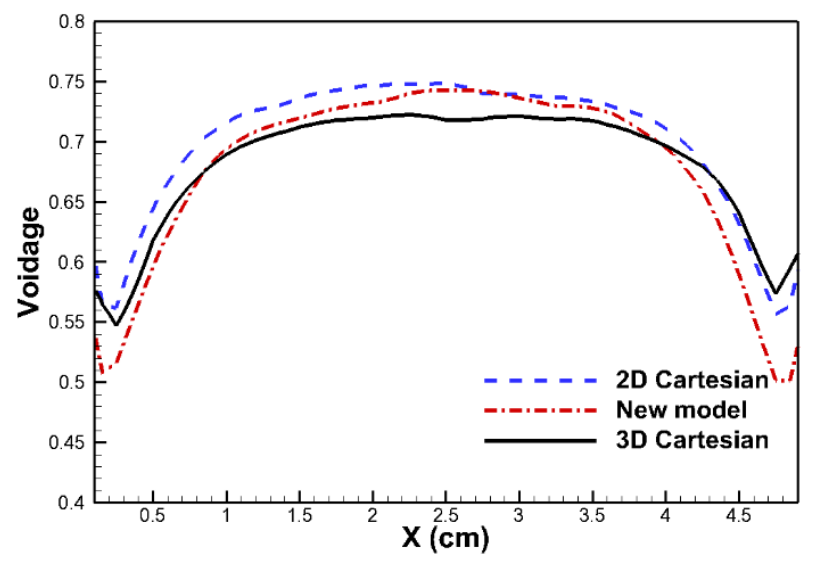

(a)

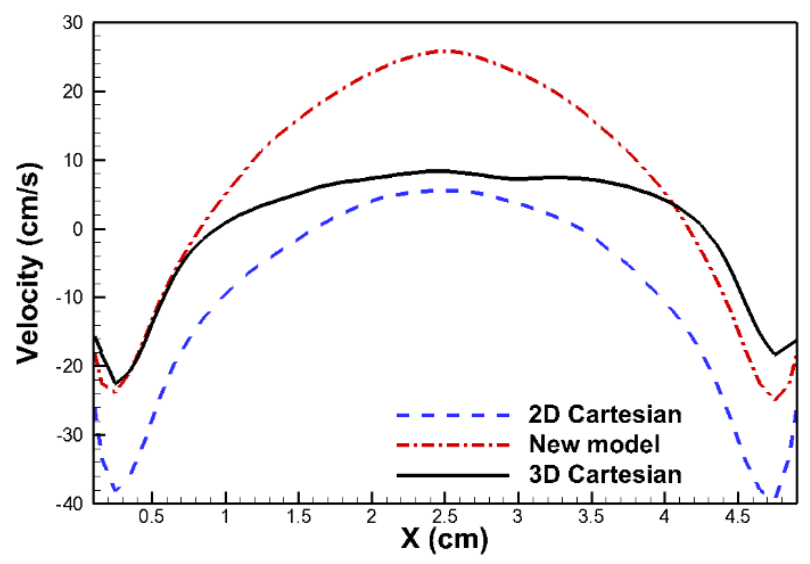

(b)

Figure 14. Radial profiles of (a) voidage and (b) vertical solids velocity at the height of $15 \mathrm{~cm}$ predicted by different simulations for the case with superficial gas velocity of $0.25 \mathrm{~m} / \mathrm{s}(\mathrm{L} / \mathrm{R}=0.28$ for the $2.5 \mathrm{D}$ model). 

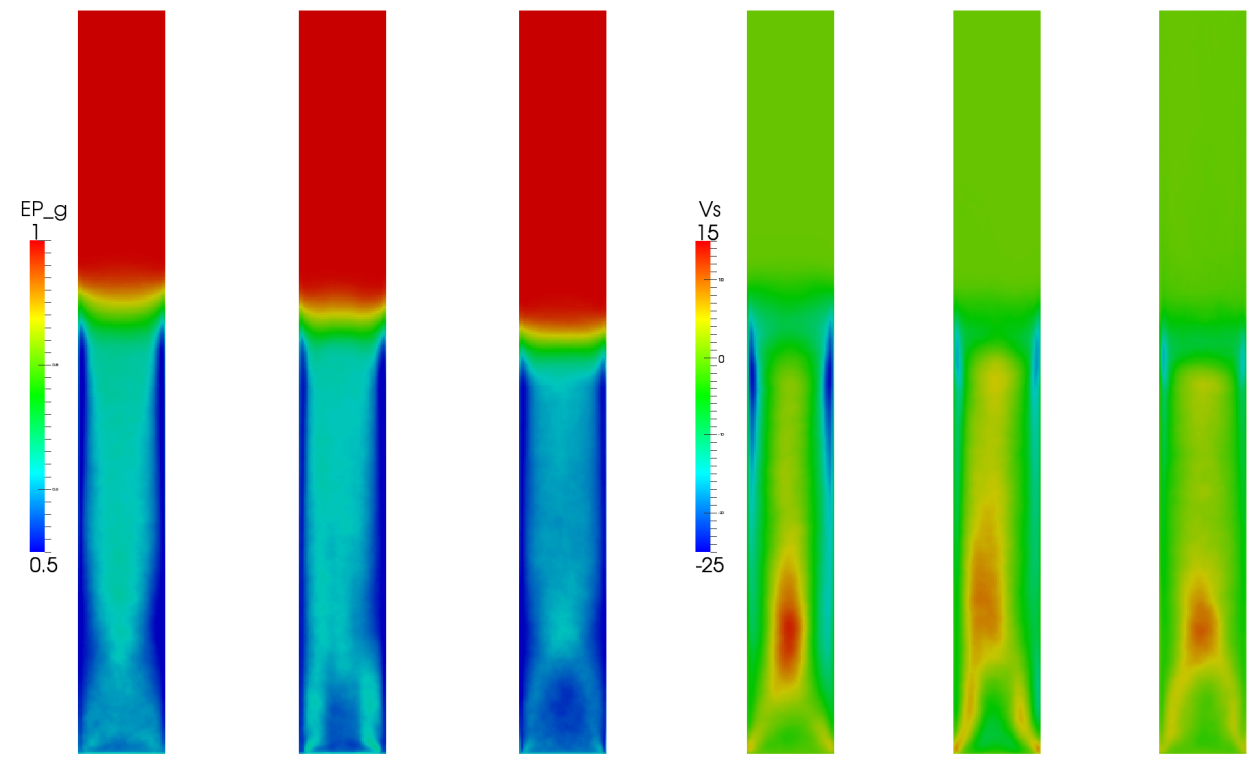

(a)
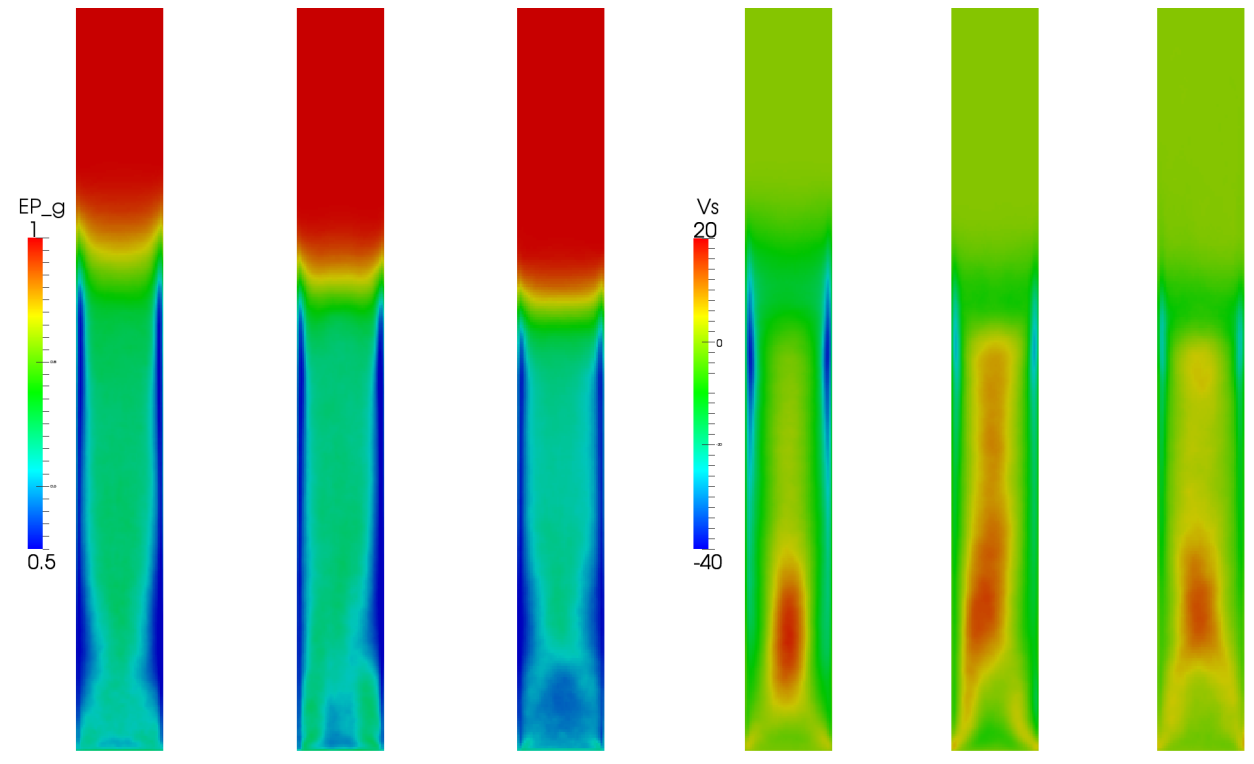

(b) 